\title{
The novel association between red complex of oral microbe and body mass index in healthy Japanese: a population based cross-sectional study
}

\author{
Kanae Matsushita, ${ }^{1}$ Masahide Hamaguchi, ${ }^{1 * *}$ Motomu Hashimoto, ${ }^{2}$ Masahiro Yamazaki, ${ }^{1}$ Toru Yamazaki, ${ }^{3}$ Keita Asai, $^{3}$ \\ Masashi Yamori, ${ }^{3}$ Kazuhisa Bessho, ${ }^{3}$ Hitoshi Toda, ${ }^{4}$ Goji Hasegawa, ${ }^{5}$ Naoto Nakamura ${ }^{1}$ and Michiaki Fukui ${ }^{1}$ \\ ${ }^{1}$ Department of Endocrinology and Metabolism, Kyoto Prefectural University of Medicine, Graduate School of Medical Science, \\ 465 Kajii-cho, Kawaramachi-Hirokoji, Kamigyo-ku, Kyoto 602-8566, Japan \\ ${ }^{2}$ Department of the Control for Rheumatic Diseases and ${ }^{3}$ Department of Oral and Maxillofacial Surgery, Graduate School of Medicine, Kyoto University, \\ 54 Kawara-cho, Shougoin, Sakyo-ku, Kyoto 606-8507, Japan \\ ${ }^{4}$ Health Checkup Center, Oike Clinic, 11 Nishino-Kyo, Shimoai-cho, Nakagyo-ku, Kyoto 604-8431, Japan \\ ${ }^{5}$ Division of Metabolism, Nephrology and Rheumatology, Japanese Red Cross Kyoto Daini Hospital, 355-5 Haruobi-cho, Kamigyo-ku, Kyoto 602-8031, Japan
}

(Received 4 February, 2015; Accepted 16 February, 2015; Published online 30 June, 2015)

\begin{abstract}
Microbiota has been thought to be one of important environmental factors for obesity or Type 2 diabetes mellitus. Among oral microbe, Porphyromonas gingivalis, Treponema denticola and Tannellera forsythia are known as risk factors, so called red complex, for periodontitis. Red complex could also be a risk factor for obesity. However, recent study indicated that obesity was not improved by periodontal therapy. Thus, we performed a cross sectional study to reveal the association of oral microbe with body mass index in a healthy population. Healthy individuals were randomly recruited. The infections of oral microbe were identified by Taqman polymerase chain reaction. The relationships between number of red complex and body mass index or waist circumference were analyzed. Two hundred and twenty-two apparently healthy Japanese were enrolled. BMI and waist circumference as well as age, periodontitis, number of brushing teeth were significantly associated with the number of red complex after adjusting covariance. The effect size of body mass index or waist circumference was $0.023(p=0.028)$ or $0.024(p=0.024)$, respectively. Body mass index and waist circumference were independently associated with the number of red complex among apparently healthy Japanese. The current observation implies the possibility that oral microbe was associated with obesity in healthy population.
\end{abstract}

Key Words: red complex, oral microbe, body mass index, waist circumference, obesity

$\mathrm{R}$ ecently several studies had reported the association between periodontitis and type 2 diabetes. $^{(1-7)}$ In spite of the positive association, the causal relationship has been controversial. Some researchers have been considered that periodontitis could be the risk factor of the development of type 2 diabetes. ${ }^{(1-5)}$ On the other hands, other researchers have been considered that diabetes could be the cause for the development of periodontitis. ${ }^{(6,7)}$ As the alternative point of view, both diseases could affect each other. As the evidence for the former, the incidence of type 2 diabetes was higher in subjects with periodontitis ${ }^{(1)}$ and glucose metabolism was impaired in them..$^{(2-5)}$ Similarly, periodontitis developed among obese subjects ${ }^{(8)}$ who were indicated the association with diabetes. Conversely, body weight was gained in subjects with periodontitis. ${ }^{(9)}$ However, it has not been clarified whether the association of periodontitis with diabetes or obesity was direct or indirect.

Although many studies indicated the positive relationship between diabetes and periodontitis as above mentioned, recent randomized controlled study indicated that the level of glucose control was not improved by periodontal therapy. ${ }^{(10)}$ In that study five hundred and fourteen type 2 diabetes patients were divided randomly into the periodontal treatment group $(n=257)$ or the control group $(n=257) .{ }^{(10)}$ As the results, clinical measurements of periodontitis were improved in the treatment group significantly, but the level of glucose control was not improved. ${ }^{(10)}$ In the same way, recent study indicated that BMI was not significantly decreased by periodontal therapy. ${ }^{(11)}$ However, there have been some unclarified facts underlying between periodontitis and diabetes or obesity. First, whole analysis of oral flora, which could affect the glucose metabolism or obesity, has not been evaluated yet. Second it has not been clarified whether complete eradication of periodontal pathogens, beyond the therapy for the periodontitis, could improve glucose control in diabetic patients and reduce body weight or not.

As described above, the relationships between periodontal pathogens and glucose metabolism or obesity are ambiguous, even the relationships between periodontitis and diabetes or obesity are exist. Nowadays, over seven hundred species have been known as oral microbe (HOMD, http://www.homd.org/). Especially, Porphyromonas gingivalis, Treponema denticola and Tannellera forsythia were more frequently detected from oral cavity of patients with periodontitis. ${ }^{(12)}$ These three species have been called as red complex, and they have been strongly related to periodontitis. ${ }^{(13)}$ Although red complex was frequently detected in diabetes patients and obese subjects, ${ }^{(14)}$ it has not been clarified whether the associations was direct or indirect. Additionally, the causal relationships have neither clarified.

To clarify the effect of obesity or impaired glucose metabolism on red complex, we performed a cross-sectional study among healthy population. In this study, we evaluated red complex, $P$. gingivalis, $T$. denticola and $T$. forsythia using Taqman real-time polymerase chain reaction (PCR) from swab samples collected

To whom correspondence should be addressed.

E-mail: seele@koto.kpu-m.ac.jp 
from buccal mucosa of Japanese healthy subjects. Glucose metabolism was evaluated by fasting blood glucose, HbAlc, immunoreactive insulin (IRI) and calculated homeostasis model assessment as an index of insulin resistance (HOMA-IR).

\section{Materials and Methods}

Study populations. Subjects were recruited randomly at the health checkup center in Kyoto, Japan from April 2013 to November 2013 after obtaining informed consent. Exclusion criteria were as follows; medication for diabetic disease, less than ten teeth, pregnancy, malignant disease, current smoker, or receiving antibiotics, dental therapy, insulin therapy, or steroidal therapy. ${ }^{(15)}$ This study was approved by the ethics committee of Kyoto Prefectural University of Medicine.

Data collection. The lifestyle factors including smoking status, habit of brushing teeth, the last time of brushing teeth and certain symptoms of oral cavity were surveyed by a standardized self-administered questionnaire. ${ }^{(16)}$ Blood-cell counts and blood chemistry were tested during the fasting state. BMI, which is the ratio of body weight $(\mathrm{kg})$ to body height $(\mathrm{m})$ squared was calculated and waist circumference was measured. ${ }^{(17)}$

Identification of three species of oral microbe from oral swab. The suitable settings for collecting swab samples were validated previously (submitting data). The detected oral microbe from bilateral buccal mucosa swab was paralleled with those from gingival sulcus. Briefly, swab samples were collected from bilateral buccal mucosa from 8 to 11 am under the following condition; in the fasting state, after brushing teeth and without using mouth rinse in the morning. The collected swabs were encapsulated in the sterile plastic tubes and conserved by dry ice. Those sterile plastic tubes were served in the $-80^{\circ} \mathrm{C}$ refrigerator quickly. Microbial DNAs were extracted from the swabs by using NucleoSpin ${ }^{\circledR}$ Tissue XS (MACHEREY-NAGEL, Düren, Germany) according to the manufacturer's procedure. The existences of specific microbial DNA were evaluated by Taqman real-time polymerase chain reaction (PCR). Taqman ${ }^{\circledR}$ Genotyping Master Mix (Applied Biosystems, Carlsbad, California) and StepOne plus Real-Time PCR System (Applied Biosystems) were used according to the preferred method of manufacture. The primers and probes for detecting T. denticola, $T$. forsythia, or P. gingivalis, were listed in Table 1 (18-21) $^{-10}$

Diagnosis of periodontal disease. We evaluated the periodontitis using with Periocatcher ${ }^{\circledR}$ (Ikagaku Co., Ltd., Kyoto, Japan). Periocatcher ${ }^{\circledR}$ is a test method for periodontitis by measuring the level of alpha 1 antitrypsin $(\alpha 1 \mathrm{AT})$ and lactoferrin (Lf) in gingival crevicular fluids (GCF). In the presence of inflammation of gingival cavity, $\alpha 1 \mathrm{AT}$ and Lf were released from gingival inflamed sites into gingival crevicular fluids. Thus, $\alpha 1 \mathrm{AT}$ and $\mathrm{Lf}$ in GCF were detected in patients with periodontitis. ${ }^{(22,23)}$ In this study, we defined periodontitis in subjects in whom both $\alpha 1 \mathrm{AT}$ and Lf were positive. The collection of GCF and the measurements of $\alpha 1 \mathrm{AT}$ and Lf were performed according to manufactures procedure. Briefly, GCF was collected from the subjects by using the unique brush. The levels of $\alpha 1 \mathrm{AT}$ and Lf in the collected GCF were quantified by enzyme-linked immunosorbent assay. The level of two standard deviation (2SD) above the mean values among healthy adults was defined as the cut off level. In the preliminary study, the average $+2 \mathrm{SD}$ of $\alpha 1 \mathrm{AT}$ and Lf among
156 healthy adults without periodontitis were $0.64 \mu \mathrm{g} / \mathrm{ml}$ and $0.83 \mu \mathrm{g} / \mathrm{ml}$ (unpublished data). Following the results, periodontitis was defined in case of both $\alpha 1 \mathrm{AT}>0.8 \mu \mathrm{g} / \mathrm{ml}$ and $\mathrm{Lf}>0.8 \mu \mathrm{g} / \mathrm{ml}$ in this study.

Statistical analysis. The SPSS statistical package, ver. 21 (SPSS, Inc., Chicago, IL) was used for all the statistical analyses and $p$ value less than 0.05 was considered statistically significant. Continuous variables among four groups were assessed by one-way analysis of variance (ANOVA), and those between two groups were assessed by $t$ test. Tukey honestly significant difference (HSD) test is used as post hoc test. The categorical values between two groups were assessed by Chi-square test. Multivariate analysis of covariance was used to analyze the effect size for the number of components of the red complex. The parameters for glucose metabolism or obesity and sex, age, smoking states, periodontitis and number of brushing teeth in a day were selected as covariance. We assessed fasting plasma glucose, immunoreactive insulin, homeostasis model assessment ratio, or HbAlc as parameters for glucose metabolism. Because the effect sizes of them among healthy population were unknown, a formal sample size estimate was not made a priori. Therefore, we set practically the sample size over two hundred before the study. Data were expressed as mean \pm SD for continuous variables and percentages (numbers) for categorical variables.

\section{Results}

Baseline characteristics of participants. Two hundred and twenty-two subjects were enrolled in this study. Age, FPG, or BMI were $52.0 \pm 11.2$ years old, $4.6 \pm 9.3 \mathrm{mg} / \mathrm{dl}$ or $22.1 \pm 3.0 \mathrm{~kg} / \mathrm{m}^{2}$ (Table 2). The positive prevalence of $P$. gingivalis, $T$. denticola, T. forsythia were $46.8 \%, 58.6 \%, 73.9 \%$, and number of components of red complex was $1.79 \pm 1.09$ (Table 2).

Univariate relationship of red complex with age, glucose metabolism, obesity, or periodontitis. At first, we applied univariate analysis to investigate the relationship of the number

Table 2. Baseline characteristics of the study subjects

\begin{tabular}{lc} 
N & \multicolumn{1}{c}{222} \\
Gender, Male \% $(\mathrm{n})$ & $65.8(146)$ \\
Age, year & $52.0 \pm 11.2$ \\
$\mathrm{FPG}, \mathrm{mg} / \mathrm{dl}$ & $94.6 \pm 9.3$ \\
$\mathrm{IRI}, \mu \mathrm{U} / \mathrm{ml}$ & $3.12 \pm 2.16$ \\
$\mathrm{HOMA}-\mathrm{R}$ & $0.74 \pm 0.56$ \\
$\mathrm{HbA} 1 \mathrm{c}, \%$ & $5.41 \pm 0.28$ \\
$\mathrm{BMI}, \mathrm{kg} / \mathrm{m}^{2}$ & $22.1 \pm 3.0$ \\
Waist circumstance, cm & $79.0 \pm 8.9$ \\
Ex-smoker, \% (n) & $16.7 \%(37)$ \\
Number of brushing teeth in a day & $2.17 \pm 0.75$ \\
Periodontal disease, \% ( $\mathrm{n})$ & $50.9 \%(113)$ \\
Positive number of red complex & $1.79 \pm 1.09$ \\
P. gingivalis, \% (n) & $46.8 \%(104)$ \\
T. denticola, \% $(\mathrm{n})$ & $58.6 \%(130)$ \\
T. forsythia, \% (n) & $73.9 \%(164)$
\end{tabular}

Continuous value are expressed as mean \pm SD, categorical values are expressed as \% (n). Abbreviations are used as fasting plasma glucose, FPG; immune reactive insulin, IRI; homeostasis model assessment ratio, HOMA-R; body mass index, BMI.

Table 1. The primer sequences for detecting indicated oral bacteria

\begin{tabular}{llll}
\hline & \multicolumn{1}{c}{ FAM labeled probe } & \multicolumn{1}{c}{ Forward primer } & \multicolumn{1}{c}{ Reverse primer } \\
\hline P. gingivalis & AGCTGTAAGATAGGCATGCGTCCCATTAGCTA & TGCAACTTGCCTTACAGAGGG & ACTCGTATCGCCCGTTATTC \\
$T$. forsythia & TGAGTAACGCGTATGTAACCTGCCCGC & AGCGATGGTAGCAATACCTGTC & TTCGCCGGGTTATCCCTC \\
$T$. denticola & ATGGGCCCGCGTCCCATTAGC & CCGAATGTGCTCATTTACATAAAGGT & GATACCCATCGTTGCCTTGGT \\
\hline
\end{tabular}


Table 3. The univariate analysis of age, metabolic parameters, BMI, waist circumferences for red complex

\begin{tabular}{|c|c|c|c|c|c|c|c|}
\hline & \multicolumn{4}{|c|}{ Positive number of infection of red complex } & \multirow{2}{*}{$\begin{array}{c}p \text { value } \\
0 \text { vs } 1\end{array}$} & \multirow{2}{*}{$\begin{array}{c}p \text { value } \\
0 \text { vs } 2\end{array}$} & \multirow{2}{*}{$\begin{array}{c}p \text { value } \\
0 \text { vs } 3\end{array}$} \\
\hline & 0 & 1 & 2 & 3 & & & \\
\hline$n$ & 36 & 51 & 58 & 77 & & & \\
\hline Age, year & $49.8 \pm 10.4$ & $49.6 \pm 11.0$ & $52.2 \pm 10.7$ & $54.5 \pm 11.7$ & 1.00 & 0.72 & 0.15 \\
\hline $\mathrm{FPG}, \mathrm{mg} / \mathrm{dl}$ & $92.1 \pm 8.7$ & $94.5 \pm 7.2$ & $96.9 \pm 10.8$ & $93.9 \pm 9.1$ & 0.63 & 0.07 & 0.77 \\
\hline IRI, $\mu \mathrm{U} / \mathrm{ml}$ & $3.2 \pm 2.9$ & $3.2 \pm 1.6$ & $3.1 \pm 2.2$ & $3.0 \pm 2.1$ & 0.99 & 0.99 & 0.98 \\
\hline HOMA-R & $0.7 \pm 0.7$ & $0.8 \pm 0.4$ & $0.8 \pm 0.6$ & $0.7 \pm 0.5$ & 0.99 & 0.99 & 0.99 \\
\hline $\mathrm{HbA} 1 \mathrm{c}, \%$ & $5.4 \pm 0.3$ & $5.3 \pm 0.3$ & $5.5 \pm 0.3$ & $5.4 \pm 0.3$ & 0.48 & 0.48 & 1.00 \\
\hline $\mathrm{BMI}, \mathrm{kg} / \mathrm{m}^{2}$ & $21.2 \pm 3.0$ & $21.8 \pm 3.2$ & $22.5 \pm 3.2$ & $22.5 \pm 2.8$ & 0.78 & 0.20 & 0.17 \\
\hline $\mathrm{WC}, \mathrm{cm}$ & $76.6 \pm 8.0$ & $77.0 \pm 8.0$ & $80.8 \pm 9.5$ & $80.0 \pm 8.9$ & 0.99 & 0.10 & 0.20 \\
\hline Brushing teeth & $2.25 \pm 0.73$ & $2.35 \pm 0.79$ & $2.10 \pm 0.76$ & $2.05 \pm 0.71$ & 0.92 & 0.79 & 0.55 \\
\hline Periodontitis & $36.1 \%(13)$ & $41.2 \%(21)$ & $56.9 \%(33)$ & $59.7 \%(46)$ & 0.79 & 0.08 & 0.03 \\
\hline Ex-smoker & $11.1 \%(4)$ & $15.7 \%(8)$ & $22.4 \%(13)$ & $15.6 \%(12)$ & 0.76 & 0.26 & 0.72 \\
\hline
\end{tabular}

Continuous values are expressed as mean \pm SD and are analyzed using with one-way analysis of variance. Tukey honestly significant difference test is used as post hoc test. Abbreviations are used as fasting plasma glucose, FPG; immune reactive insulin, IRI; homeostasis model assessment ratio, HOMA-R; body mass index, BMI; waist circumferences, WC. Red complex is consisted with P. gingivalis, T. denticola and T. forsythia.

Table 4. The univariate analysis of age, metabolic parameters, BMI, and waist circumferences for $P$. gingivalis, T. denticola or T. forsythia

\begin{tabular}{|c|c|c|c|c|c|c|c|c|c|}
\hline \multirow[b]{3}{*}{$n$} & \multicolumn{3}{|c|}{ P. gingivalis } & \multicolumn{3}{|c|}{ T. denticola } & \multicolumn{3}{|c|}{ T. forsythia } \\
\hline & Negative & Positive & \multirow{2}{*}{$p$ value } & Negative & Positive & \multirow{2}{*}{$p$ value } & Negative & Positive & \multirow{2}{*}{$p$ value } \\
\hline & 118 & 104 & & 92 & 130 & & 58 & 164 & \\
\hline Age, year & $49.4 \pm 10.4$ & $55 \pm 11.4$ & $<0.001$ & $51.2 \pm 11.1$ & $52.6 \pm 11.2$ & 0.38 & $50.3 \pm 10.3$ & $52.6 \pm 11.4$ & 0.18 \\
\hline FPG, mg/dl & $94.7 \pm 9.4$ & $94.4 \pm 9.2$ & 0.76 & $94.1 \pm 8.8$ & $94.9 \pm 9.6$ & 0.49 & $92.9 \pm 7.9$ & $95.2 \pm 9.6$ & 0.10 \\
\hline IRI, $\mu \mathrm{U} / \mathrm{ml}$ & $3.16 \pm 2.2$ & $3.08 \pm 2.12$ & 0.76 & $3.21 \pm 2.34$ & $3.06 \pm 2.04$ & 0.60 & $3.22 \pm 2.44$ & $3.09 \pm 2.06$ & 0.67 \\
\hline HOMAR & $0.76 \pm 0.57$ & $0.73 \pm 0.56$ & 0.76 & $0.76 \pm 0.6$ & $0.73 \pm 0.53$ & 0.68 & $0.75 \pm 0.6$ & $0.74 \pm 0.55$ & 0.92 \\
\hline HbA1c, \% & $5.42 \pm 0.29$ & $5.41 \pm 0.27$ & 0.87 & $5.38 \pm 0.28$ & $5.44 \pm 0.29$ & 0.10 & $5.39 \pm 0.29$ & $5.42 \pm 0.28$ & 0.44 \\
\hline BMI, kg/m² & $21.7 \pm 3.2$ & $22.6 \pm 2.9$ & 0.038 & $21.9 \pm 3.3$ & $22.3 \pm 2.9$ & 0.31 & $21.5 \pm 2.9$ & $22.4 \pm 3.1$ & 0.063 \\
\hline $\mathrm{WC}, \mathrm{cm}$ & $77.5 \pm 8.6$ & $80.6 \pm 8.9$ & 0.009 & $78 \pm 8.4$ & $79.6 \pm 9.1$ & 0.19 & $77.2 \pm 8.2$ & $79.6 \pm 9$ & 0.081 \\
\hline Brushing teeth & $2.16 \pm 0.76$ & $2.17 \pm 0.74$ & 0.95 & $2.29 \pm 0.76$ & $2.08 \pm 0.73$ & 0.034 & $2.38 \pm 0.74$ & $2.09 \pm 0.74$ & 0.012 \\
\hline Periodontitis & $46.6 \%(55)$ & $55.8 \%(58)$ & 0.18 & $41.3 \%(38)$ & $57.7 \%(75)$ & 0.02 & $36.2 \%(21)$ & $56.1 \%(92)$ & 0.01 \\
\hline Ex-smoker & $16.1 \%$ (19) & $17.3 \%(18)$ & 0.86 & $15.2 \%(14)$ & $17.7 \%(23)$ & 0.72 & $13.8 \%(8)$ & $17.7 \%(29)$ & 0.55 \\
\hline
\end{tabular}

Continuous values are expressed as mean \pm SD and are analyzed using with one-way analysis of variance. Tukey honestly significant difference test is used as post hoc test. Abbreviations are used as fasting plasma glucose, FPG; immune reactive insulin, IRI; homeostasis model assessment ratio, HOMA-R; body mass index, BMI; waist circumferences, WC.

of components of red complex with age, glucose metabolism, obesity, or periodontitis. For these purposes, we applied analysis of variance and post hoc test. The proportions of periodontitis were significantly higher in subjects with 2 or 3 of components of red complex. However, FPG, HOMA-R, and HbAlc were equal levels among 4 grades of components of red complex. Interestingly, BMI and waist circumferences were higher in subjects with 2 or 3 of components of red complex, but they were not statistically significant. On the other hands, IRI was low in subjects with 2 or 3 of components of red complex, but it was neither statistically significant (Table 3 ).

Next, we assessed the relationship of the existence of $P$. gingivalis, $T$. denticola, or $T$. forsythia with age, glucose metabolism, obesity, or periodontitis. Interestingly, periodontitis was higher in subjects with $T$. denticola, or $T$. forsythia, and the number of brushing teeth in a day was lower in subjects with $T$. denticola, or T. forsythia. On the other hand, age was higher in subjects with $P$. gingivalis (Table 4). BMI and waist circumferences were significantly higher in subjects with $P$. gingivalis ( $p=0.038$ and $p=0.009$, respectively). In the same way, BMI and waist circumferences were significantly higher in subjects with $T$. denticola, or $T$. forsythia, but they were not statistically significant (Table 4).

The adjusted effect size for the number of red complex. To clarify direct relationship of the number of components of red complex with BMI or waist circumferences, we applied multi- variate analysis of covariance and calculate the effect size of each BMI or waist circumferences for the number of components of red complex after adjusting FPG, IRI, sex, age, smoking states, periodontitis, the number of brushing teeth in a day. As results, statistically significant relationships were observed between the number of components of red complex and BMI, waist circumferences, age, periodontitis or the number of brushing teeth in a day (Table 5). The effect size of BMI or waist circumferences was $0.023(p=0.028)$, or $0.024(p=0.024)$, respectively. However, the effect sizes of FPG was strikingly low as 0.00 .

\section{Discussion}

The current study clearly demonstrated that both BMI and waist circumferences were associated with the number of red complex in apparently healthy Japanese population independently on the other covariance. Previously, it was reported that body weight was gained in subjects with periodontitis. ${ }^{(9)}$ In addition to this evidence, our findings indicated that the number of red complex was higher in subjects with high BMI or waist circumferences independent on periodontitis. And more, it was also reported that the incidence of periodontitis was higher among obese subjects. ${ }^{(8)}$ Our research implies the possible reason of this evidence. Because the number of red complex was high in obese individuals even if they were not diagnosed as periodontitis, they would be easy to be suffered from periodontitis. Additionally, the current study showed 
Table 5. The adjusted effect size for the number of red complex

\begin{tabular}{lcclcc}
\hline & $p$ & Effect size & & $p$ & Effect size \\
\hline FPG, mg/dl & 0.960 & 0.000 & FPG, mg/dl & 0.805 & 0.000 \\
IRI, $\mu \mathrm{U} / \mathrm{ml}$ & 0.083 & 0.014 & IRI, $\mu \mathrm{U} / \mathrm{ml}$ & 0.091 & 0.014 \\
Sex & 0.091 & 0.013 & Sex & 0.131 & 0.011 \\
Age, years & 0.023 & 0.024 & Age, years & 0.033 & 0.021 \\
Ex-smoker & 0.198 & 0.008 & Smoker & 0.246 & 0.006 \\
Periodontitis & 0.012 & 0.029 & Periodontitis & 0.010 & 0.031 \\
Brushing teeth & 0.185 & 0.008 & Brushing teeth & 0.213 & 0.007 \\
BMI, kg/m & 0.028 & 0.023 & WC, cm & 0.024 & 0.024 \\
\hline
\end{tabular}

Multivariate analysis of covariance is used for calculating the effect size of each parameter for the number of components of red complex. FPG, IRI, sex, age, smoking states, periodontitis, number of brushing teeth in a day are used as covariances. Abbreviations are used as fasting plasma glucose, FPG; immune reactive insulin, IRI; homeostasis model assessment ratio, HOMA-R; body mass index, BMl; waist circumferences, WC. Red complex is consisted with $P$. gingivalis, T. denticola and T. forsythia.

that $P$. gingivalis had strong correlation with age. Previously, Savitt et $a l .{ }^{(24)}$ and Umeda et al. ${ }^{(25)}$ also indicated the association between $P$. gingivalis and aging.

Two hypotheses for the mechanism of the relationship between diabetes and periodontitis have been thought as follows; the incidence of periodontitis was high in individuals with diabetes. Or impaired glucose metabolism would be induced in individuals with periodontitis. As the biological explanation for the former, immune reaction against bacteria were reduced in individuals with diabetes, and following that, the destruction of periodontal tissue would be tend to be induced among individuals with diabetes. ${ }^{(6,26,27)}$ In addition to that, our findings added the new inspect that red complex in individuals with both diabetes and obesity might be a risk factor for the newly onset of periodontitis.

On the other hands, as the biological explanation for the latter, kinds of the infection of oral microbe might be the resource of inflammatory cytokines. In fact, inflammatory cytokines were thought to be released at periodontal tissue by red complex. ${ }^{(28-36)}$ Insulin resistance was thought to be increased by these inflammatory cytokines. ${ }^{(37)}$ Our study indicated that the number of red complex was higher in non-diabetic individuals with high BMI or waist circumferences. This evidence implies the possibility that insulin resistance is going to be increased by both obesity and inflammatory cytokines activated by red complex.

Our study has some limitations. First, periodontitis was not diagnosed by dentist, but was evaluated by Periocatcher ${ }^{\circledR}$. Sensi-

\section{References}

1 Demmer RT, Jacobs DR Jr, Desvarieux M. Periodontal disease and incident type 2 diabetes: results from the First National Health and Nutrition Examination Survey and its epidemiologic follow-up study. Diabetes Care 2008; 31: 1373-1379.

2 Saito T, Shimazaki Y, Kiyohara Y, et al. The severity of periodontal disease is associated with the development of glucose intolerance in non-diabetics: the Hisayama study. J Dent Res 2004; 83: 485-490.

3 Collin HL, Uusitupa M, Niskanen L, et al. Periodontal findings in elderly patients with non-insulin dependent diabetes mellitus. J Periodontol 1998; 69: 962-966.

4 Demmer RT, Desvarieux M, Holtfreter B, et al. Periodontal status and A1C change: longitudinal results from the study of health in Pomerania (SHIP). Diabetes Care 2010; 33: 1037-1043.

5 Taylor GW, Burt BA, Becker MP, et al. Severe periodontitis and risk for poor glycemic control in patients with non-insulin-dependent diabetes mellitus. J Periodontol 1996; 67 (10 Suppl): 1085-1093.

6 Löe H. Periodontal disease. The sixth complication of diabetes mellitus. Diabetes Care 1993; 16: 329-334.

7 Taylor GW, Burt BA, Becker MP, et al. Non-insulin dependent diabetes mellitus and alveolar bone loss progression over 2 years. J Periodontol 1998; tivity and specificity of Periocatcher ${ }^{\circledR}$ for periodontitis were 0.7 and 0.7 respectively (unpublished data). Moreover, the proportion of periodontitis in the current study was the same as that reported in previous study. ${ }^{(38)}$ The prevalence of periodontitis was $50.9 \%$ among 222 nonsmoking subjects with mean age of 52 years in this study. That was $46.6 \%$ among 2,055 Japanese subjects who were non-smoker and whose age was ranged from 40 to $59 .^{(38)}$ Second, generalization of our findings could be limited because our study was a pilot study and subjects were consisted with Japanese.

BMI and waist circumferences were associated with the number of red complex in apparently healthy Japanese population independently on other covariance. This finding implies a novel explanation for the positive association between periodontitis and type 2 diabetes.

\section{Acknowledgments}

The authors thank all staffs in the Health Checkup Center, Oike Clinic, Kyoto, Japan. We are grateful to the participants and all of the medical staff cooperating with us. This study was supported by Young Scientists (B) from Japan Society for the Promotion of Science, No. 23790791 and 26860502.

\section{Conflict of Interest}

No potential conflicts of interest were disclosed.
69: 76-83.

8 Amar S, Zhou Q, Shaik-Dasthagirisaheb Y, Leeman S. Diet-induced obesity in mice causes changes in immune responses and bone loss manifested by bacterial challenge. Proc Natl Acad Sci US A 2007; 104: 20466-20471.

9 Morita T, Yamazaki Y, Mita A, et al. A cohort study on the association between periodontal disease and the development of metabolic syndrome. $J$ Periodontol 2010; 81: 512-519.

10 Engebretson SP, Hyman LG, Michalowicz BS, et al. The effect of nonsurgical periodontal therapy on hemoglobin A1c levels in persons with type 2 diabetes and chronic periodontitis: a randomized clinical trial. JAMA 2013; 310: $2523-2532$.

11 Zuza EP, Barroso EM, Carrareto AL, et al. The role of obesity as a modifying factor in patients undergoing non-surgical periodontal therapy. $J$ Periodontol 2011; 82: 676-682.

12 Carinci F, Scapoli L, Girardi A, et al. Oral microflora and periodontal disease: new technology for diagnosis in dentistry. Ann Stomatol (Roma) 2013; 4: 170-173.

13 Socransky SS, Haffajee AD. Periodontal microbial ecology. Periodontol 20002005 ; 38: 135-187.

14 Aemaimanan P, Amimanan P, Taweechaisupapong S. Quantification of key 
periodontal pathogens in insulin-dependent type 2 diabetic and non-diabetic patients with generalized chronic periodontitis. Anaerobe 2013; 22: 64-68.

15 Shimazaki Y, Saito T, Yonemoto K, Kiyohara Y, Iida M, Yamashita Y. Relationship of metabolic syndrome to periodontal disease in Japanese women: the Hisayama Study. J Dent Res 2007; 86: 271-275.

16 Hamaguchi M, Kojima T, Takeda N, et al. The metabolic syndrome as a predictor of nonalcoholic fatty liver disease. Ann Intern Med 2005; 143: 722728.

17 Executive summary of the clinical guidelines on the identification, evaluation, and treatment of overweight and obesity in adults. Arch Intern Med 1998; 158: $1855-1867$.

18 Nadkarni MA, Martin FE, Jacques NA, Hunter N. Determination of bacterial load by real-time PCR using a broad-range (universal) probe and primers set. Microbiology 2002; 148 (Pt 1): 257-266.

19 Kuboniwa M, Amano A, Kimura KR, et al. Quantitative detection of periodontal pathogens using real-time polymerase chain reaction with TaqMan probes. Oral Microbiol Immunol 2004; 19: 168-176.

20 Nonnenmacher C, Dalpke A, Mutters R, Heeg K. Quantitative detection of periodontopathogens by real-time PCR. J Microbiol Methods 2004; 59: 117 125.

21 Yang S, Lin S, Kelen GD, et al. Quantitative multiprobe PCR assay for simultaneous detection and identification to species level of bacterial pathogens. J Clin Microbiol 2002; 40: 3449-3454.

22 Lourenço AG, Nakao C, Machado AA, et al. Lactoferrin, a marker for periodontal disease. Curr HIV Res 2013; 11: 220-225.

23 Adonogianaki E, Moughal NA, Mooney J, Stirrups DR, Kinane DF. Acutephase proteins in gingival crevicular fluid during experimentally induced gingivitis. J Periodontal Res 1994; 29: 196-202.

24 Savitt ED, Kent RL. Distribution of Actinobacillus actinomycetemcomitans and Porphyromonas gingivalis by subject age. J Periodontol 1991; 62: 490494.

25 Umeda M, Chen C, Bakker I, Contreras A, Morrison JL, Slots J. Risk indicators for harboring periodontal pathogens. J Periodontol 1998; 69: 1111-1118.

26 Amano A, Kawai S. Diabetes and oral osteoporosis. Clin Calcium 2007; 17: 186-191 (in Japanese).

27 Shetty N, Thomas B, Ramesh A. Comparison of neutrophil functions in diabetic and healthy subjects with chronic generalized periodontitis. $J$ Indian Soc Periodontol 2008; 12: 41-44.

28 Vassiliadis S, Dragiotis V, Protopapadakis E, et al. The destructive action of IL-1alpha and IL-1beta in IDDM is a multistage process: evidence and confirmation by apoptotic studies, induction of intermediates and electron microscopy. Mediators Inflamm 1999; 8: 85-91.

29 Sjöholm A. Aspects of the involvement of interleukin-1 and nitric oxide in the pathogenesis of insulin-dependent diabetes mellitus. Cell Death Differ 1998; 5: 461-468.

30 Moller DE. Potential role of TNF-alpha in the pathogenesis of insulin resistance and type 2 diabetes. Trends Endocrinol Metab 2000; 11: 212-217.

31 Qi C, Pekala PH. Tumor necrosis factor-alpha-induced insulin resistance in adipocytes. Proc Soc Exp Biol Med 2000; 223: 128-135.

32 Gaibani P, Caroli F, Nucci C, Sambri V. Major surface protein complex of Treponema denticola induces the production of tumor necrosis factor alpha, interleukin-1 beta, interleukin- 6 and matrix metalloproteinase 9 by primary human peripheral blood monocytes. J Periodontal Res 2010; 45: 361-366.

33 Sahingur SE, Xia XJ, Alamgir S, Honma K, Sharma A, Schenkein HA. DNA from Porphyromonas gingivalis and Tannerella forsythia induce cytokine production in human monocytic cell lines. Mol Oral Microbiol 2010; 25: 123-135.

34 Nishihara R, Sugano N, Takano M, et al. The effect of Porphyromonas gingivalis infection on cytokine levels in type 2 diabetic mice. $J$ Periodontal Res 2009; 44: 305-310.

$35 \mathrm{Li} \mathrm{H}$, Yang $\mathrm{H}$, Ding $\mathrm{Y}$, et al. Experimental periodontitis induced by Porphyromonas gingivalis does not alter the onset or severity of diabetes in mice. J Periodontal Res 2013; 48: 582-590.

36 Morran MP, Alexander LA, Slotterbeck BD, McInerney MF. Dysfunctional innate immune responsiveness to Porphyromonas gingivalis lipopolysaccharide in diabetes. Oral Microbiol Immunol 2009; 24: 331-339.

37 Tilg H, Moschen AR. Insulin resistance, inflammation, and non-alcoholic fatty liver disease. Trends Endocrinol Metab 2008; 19: 371-379.

38 Ojima M, Hanioka T, Shimada K, Haresaku S, Yamamoto M, Tanaka K. The role of tobacco use on dental care and oral disease severity within community dental clinics in Japan. Tob Induc Dis 2013; 11: 13. 